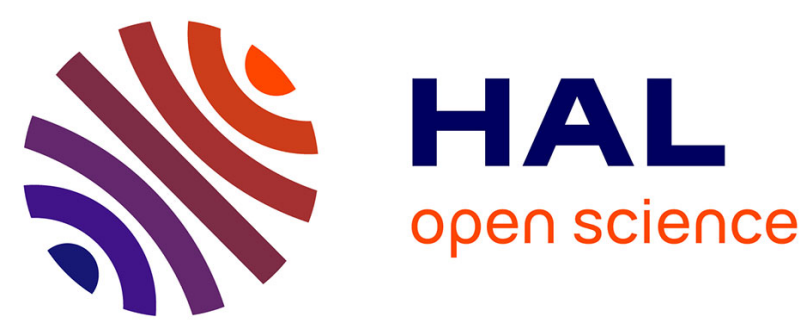

\title{
Chasing Aqueous Biphasic Systems from Simple Salts by Exploring the LiTFSI/LiCl/H2O Phase Diagram
}

Nicolas Dubouis, Chanbum Park, Michael Deschamps, Soufiane

Abdelghani-Idrissi, Matej Kanduč, Annie Colin, Mathieu Salanne, Joachim

Dzubiella, Alexis Grimaud, Benjamin Rotenberg

\section{To cite this version:}

Nicolas Dubouis, Chanbum Park, Michael Deschamps, Soufiane Abdelghani-Idrissi, Matej Kanduč, et al.. Chasing Aqueous Biphasic Systems from Simple Salts by Exploring the LiTFSI/LiCl/H2O Phase Diagram. ACS Central Science, 2019, 5 (4), pp.640-643. 10.1021/acscentsci.8b00955 . hal-02124064

\section{HAL Id: hal-02124064 https://hal.sorbonne-universite.fr/hal-02124064}

Submitted on 9 May 2019

HAL is a multi-disciplinary open access archive for the deposit and dissemination of scientific research documents, whether they are published or not. The documents may come from teaching and research institutions in France or abroad, or from public or private research centers.
L'archive ouverte pluridisciplinaire HAL, est destinée au dépôt et à la diffusion de documents scientifiques de niveau recherche, publiés ou non, émanant des établissements d'enseignement et de recherche français ou étrangers, des laboratoires publics ou privés. 


\section{Chasing Aqueous Biphasic Systems from Simple Salts by Exploring the $\mathrm{LiTFSI} / \mathrm{LiCl} / \mathrm{H}_{2} \mathrm{O}$ Phase Diagram}

Nicolas Dubouis, ${ }^{\dagger,}, \S \odot$ Chanbum Park, ${ }^{\|, \perp} \odot$ Michäl Deschamps, ${ }^{\S, \#}$ Soufiane Abdelghani-Idrissi, ${ }^{\bigcirc}$ Matej Kanduč, ${ }^{\prime \prime} @ \circledast$ Annie Colin, ${ }^{\bigcirc}$ Mathieu Salanne, ${ }^{\S, \nabla} \odot$ Joachim Dzubiella, ${ }^{*}, \|, \bullet \odot$ Alexis Grimaud, $*,+,, \S_{\odot}$ and Benjamin Rotenberg $*, \S^{*}, \nabla_{\odot}$

${ }^{\dagger}$ Chimie du Solide et de l’Energie, Collège de France, UMR 8260, 75231 Paris Cedex 05, France

${ }^{\ddagger}$ Sorbonne Université, Paris, France

${ }^{\S}$ Réseau sur le Stockage Electrochimique de l'Energie (RS2E), CNRS FR3459, 33 rue Saint Leu, 80039 Amiens Cedex, France

"Research Group for Simulations of Energy Materials, Helmholtz-Zentrum Berlin für Materialien und Energie, Hahn-Meitner-Platz 1, 14109 Berlin, Germany

${ }^{\perp}$ Institut für Physik, Humboldt-Universität zu Berlin, Newtonstrasse 15, 12489 Berlin, Germany

\#CNRS, CEMHTI UPR3079, Université d'Orléans, 1D av. de la recherche scientifique, 45071 Orléans Cedex 2, France

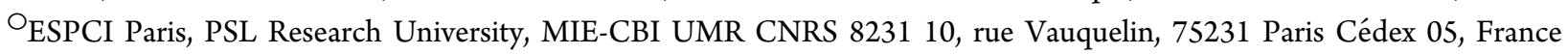

${ }^{\circledR}$ Jožef Stefan Institute, Jamova 39, 1000 Ljubljana, Slovenia

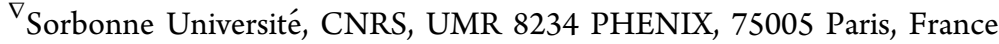

•Applied Theoretical Physics-Computational Physics, Physikalisches Institut, Albert-Ludwigs-Universität Freiburg, Hermann-Herder Strasse 3, 79104 Freiburg, Germany

Supporting Information

ABSTRACT: Aqueous biphasic systems (ABSs), in which two aqueous phases with different compositions coexist as separate liquids, were first reported more than a century ago with polymer solutions. Recent observations of ABS forming from concentrated mixtures of inorganic salts and ionic liquids raise the fundamental question of how "different" the components of such mixtures should be for a liquid-liquid phase separation to occur. Here we show that even two monovalent salts sharing a common cation (lithium) but with different anions, namely, $\mathrm{LiCl}$ and lithium bis(trifluoromethanesulfonyl)imide (LiTFSI), may result in the formation of $\mathrm{ABSs}$ over a wide range of compositions at room temperature. Using a combination of experimental techniques and molecular simulations, we analyze the coexistence diagram and the mechanism driving the phase separation, arising from the different anion sizes. The understanding and control of $\mathrm{ABS}$ may provide new avenues for aqueous-based battery systems.

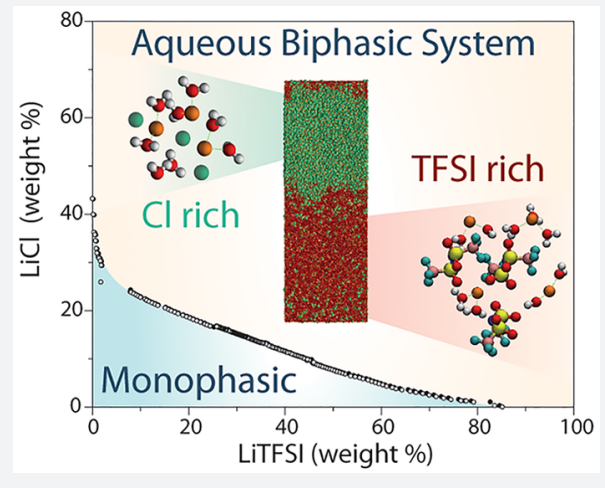

$\mathrm{T}$ he development of aqueous Li-ion batteries has recently led to the exploration of highly concentrated aqueous electrolyte solutions using organic lithium salts to slow the kinetics of water splitting in the vicinity of the electrodes and therefore enable higher operating voltages. ${ }^{1-3}$ In an attempt to decrease the amount of expensive organic salt used in these aqueous electrolytes, ${ }^{4}$ we investigated mixtures with an inexpensive lithium salt and could observe an unusual phenomenon, namely the formation of an aqueous biphasic system (ABS). When two liquids with very different physicochemical properties, such as water and oil, contact each other, they usually coexist as separate phases with a sharp interface. This fact has long been exploited, e.g., for the separation of aqueous solutes via liquid-liquid extraction using organic phases or ionic liquids. ${ }^{5-7}$ While the first ABSs based on polymers were discovered in the late 19 th century, ${ }^{8}$ the coexistence of two aqueous solutions of different salts has been reported only much more recently and the range of such ABSs remains comparatively limited. ${ }^{9-11}$ Their composition usually involves a concentrated inorganic salt and an ionic liquid. The resulting phases both contain a sizable amount of water (hence their name), and the origin of the phase separation is thought to result from the simultaneous presence of water hydrogen bond network structuring (kosmotropic) or disordering (chaotropic) ions. ${ }^{10}$ This raises the fundamental question of how "different" the components of the mixture should be for such a liquid-liquid phase separation to occur, i.e., in the aqueous case, for an ABS to form. Here we show that even mixtures of two monovalent salts sharing a common simple cation (lithium, $\mathrm{Li}^{+}$) but with different anions result in the

Received: December 20, 2018

Published: March 12, 2019 
presence of water in the coexistence of two aqueous liquid phases over a wide range of compositions at room temperature.

Specifically, we investigate aqueous mixtures of lithium bis(trifluoromethanesulfonyl)imide (LiTFSI), which can form so-called "water-in-salt"1-3 electrolytes (with a salt:solvent ratio of $>1$ in mass and volume) relevant, e.g., for battery applications, and the more standard lithium chloride $(\mathrm{LiCl})$ salt (see Figure 1A). We show in Figure 1B that a global
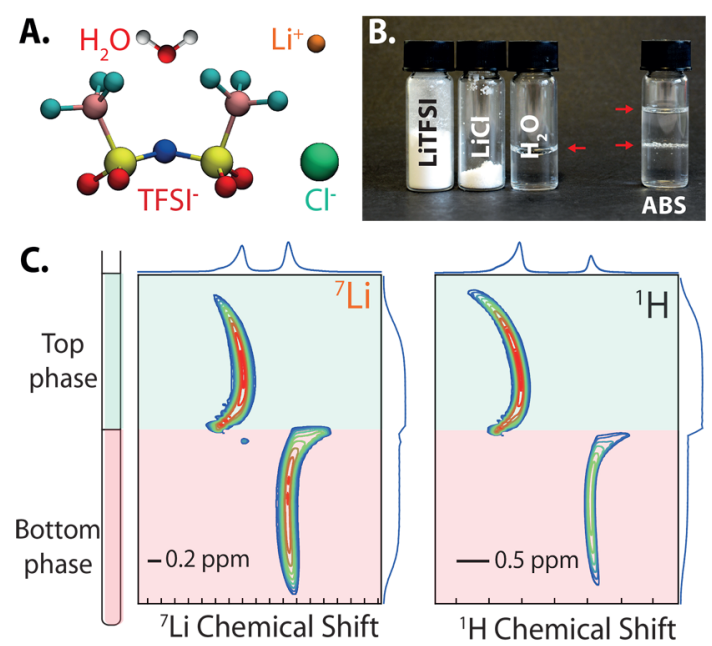

D.

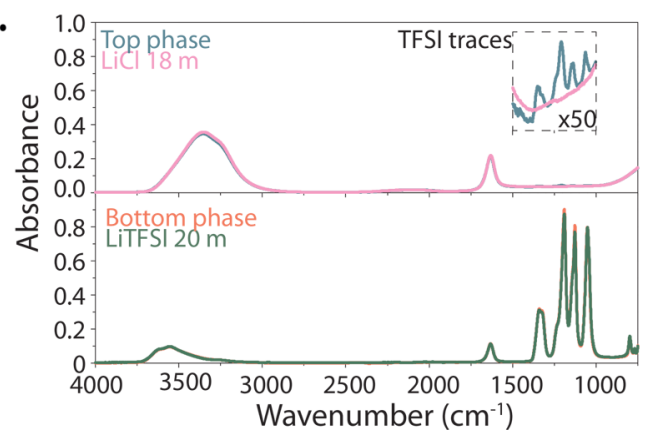

Figure 1. (A) Constituents of the system: water molecule, $\mathrm{Li}^{+}$cation, TFSI $^{-}$and $\mathrm{Cl}^{-}$anions. (B) Stoichiometric amount of LiTFSI, LiCl, and water used to prepare the $12 \mathrm{~m} \mathrm{LiCl}-5 \mathrm{~m}$ LiTFSI aqueous biphasic system (ABS). (C) Chemical shift imaging (CSI) of a nuclear magnetic resonance tube containing the $12 \mathrm{~m} \mathrm{LiCl}-5 \mathrm{~m}$ LiTFSI ABS, revealing distinct local environments for $\mathrm{Li}^{+}$cations and water in the two phases. The color indicates the intensity (from blue for low to red for high) as a function of chemical shift and position, while the shape of the peaks reflects the distortion of the magnetic field near the liquid-liquid and liquid-air interfaces. (D) Fourier transform infrared spectra of the top and bottom phases of the $12 \mathrm{~m}$ LiCl-5m LiTFSI ABS compared with those of an $18 \mathrm{~m} \mathrm{LiCl}$ solution and a $20 \mathrm{~m}$ LiTFSI solution.

composition of $12 \mathrm{~m}$ (moles per kilogram of water) $\mathrm{LiCl}$ and $5 \mathrm{~m}$ LiTFSI results in approximately equal volumes of two coexisting liquids with a sharp interface. The formation of an interface between these two liquids exchanging matter is further evidenced in the Supporting Information by images showing the evolution of a drop of $5 \mathrm{~m}$ LiTFSI in a saturated $(18 \mathrm{~m}) \mathrm{LiCl}$ solution as well as by the measurement of a finite surface tension $(5.7 \mathrm{mN} / \mathrm{m}$ for the $12 \mathrm{~m} \mathrm{LiCl}-5 \mathrm{~m}$ LiTFSI, approximately an order of magnitude smaller than for the water-air interface).

Nuclear magnetic resonance (NMR) then provides spatially resolved specific information about the chemical environment of the various species. The evolution of the chemical shifts across the interface (Figure 1C) indicates that both $\mathrm{Li}^{+}$cations and water molecules adopt distinct local environments in the two liquid phases, while the change in the dielectric constant results in a distortion at the interface between the two phases. In addition, Fourier transform infrared (FTIR) spectroscopy allows us to probe the local environment of water in both phases, which in turn provides indirect information about their composition. As shown in Figure 1D, water molecules from the top phase exhibit $\mathrm{O}-\mathrm{H}$ bond stretching modes (broad signal around $3400 \mathrm{~cm}^{-1}$ ) similar to those in an aqueous $18 \mathrm{~m} \mathrm{LiCl}$ solution, while weak absorbance peaks around $1250 \mathrm{~cm}^{-1}$ reveal traces of TFSI in solution. ${ }^{12} \mathrm{~A}$ similar resemblance between the bottom phase and aqueous $20 \mathrm{~m}$ LiTFSI can also be deduced from their FTIR spectra.

The liquid-liquid phase separation is further quantitatively examined in Figure 2, which compares the equilibrium density

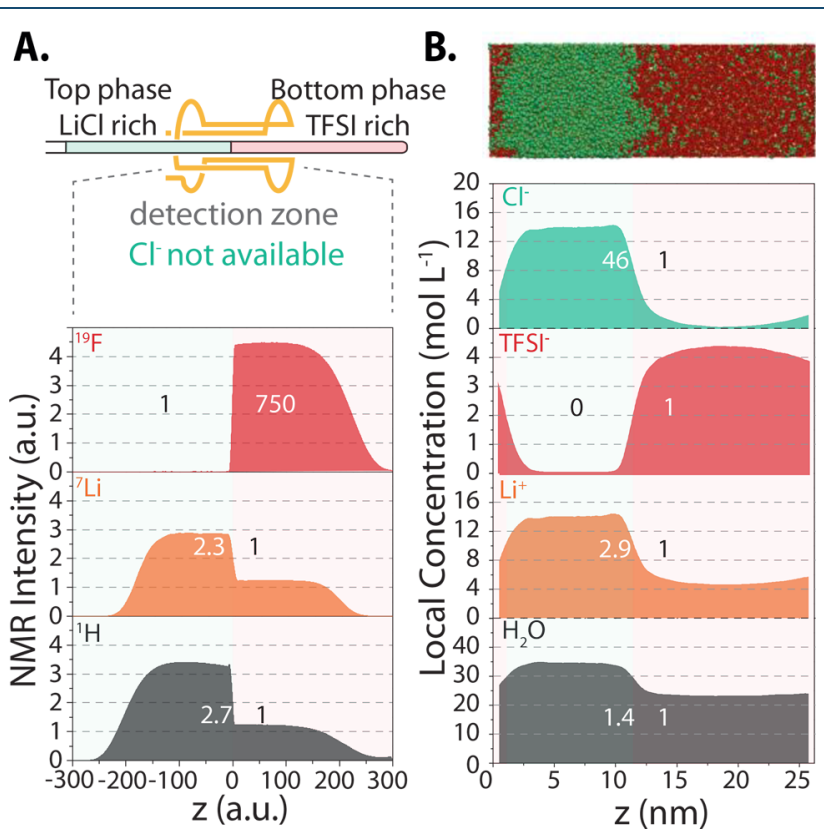

Figure 2. (A) Density profiles for ${ }^{19} \mathrm{~F},{ }^{7} \mathrm{Li}$, and ${ }^{1} \mathrm{H}$ obtained by NMR for the $12 \mathrm{~m} \mathrm{LiCl}-5 \mathrm{~m} \mathrm{LiCl} \mathrm{ABS}$ compared with (B) those obtained by molecular dynamics simulations for $\mathrm{Cl}^{-}, \mathrm{TFSI}^{-}, \mathrm{Li}^{+}$, and $\mathrm{H}_{2} \mathrm{O}$ for the same system. On the top of panel $\mathrm{B}$, a snapshot extracted from the simulation $\left(\mathrm{Li}^{+}\right.$ions are colored orange, $\mathrm{Cl}^{-}$anions green, $\mathrm{TFSI}^{-}$ anions red, and water molecules are not shown) illustrates the presence of a sharp interface.

profiles for various elements probed by NMR imaging experiments and computed by molecular dynamics (MD) simulations (see Methods). Despite the difference in length scales probed by both approaches $(\sim 1 \mathrm{~cm}$ for NMR and $\sim 10$ $\mathrm{nm}$ for $\mathrm{MD}$ ), they provide a consistent picture of a sharp interface between two coexisting liquids. The compositions are in good agreement for the species that we could probe experimentally (all but $\mathrm{Cl}^{-}$anions), which validates the $\mathrm{MD}$ simulations. In particular, MD simulations predict almost quantitatively the ratio of $\mathrm{Li}^{+}$concentration in both phases as well as the virtual absence of TFSI $^{-}$in the Cl-rich phase. The predicted water content in the TFSI-rich phase is smaller than in the other, even though the ratio is slightly higher than the experimental one. The density profile for $\mathrm{Cl}^{-}$is also readily available from $\mathrm{MD}$ and indicates that its concentration in the 
TFSI-rich phase is higher than that of $\mathrm{TFSI}^{-}$in the Cl-rich phase.

The formation of an ABS, i.e., phase separation between two liquid phases, depends on the global composition of the system. The boundary between the monophasic and biphasic regions of the phase diagram, determined by cloud point measurements (see Methods), is shown in Figure 3. With

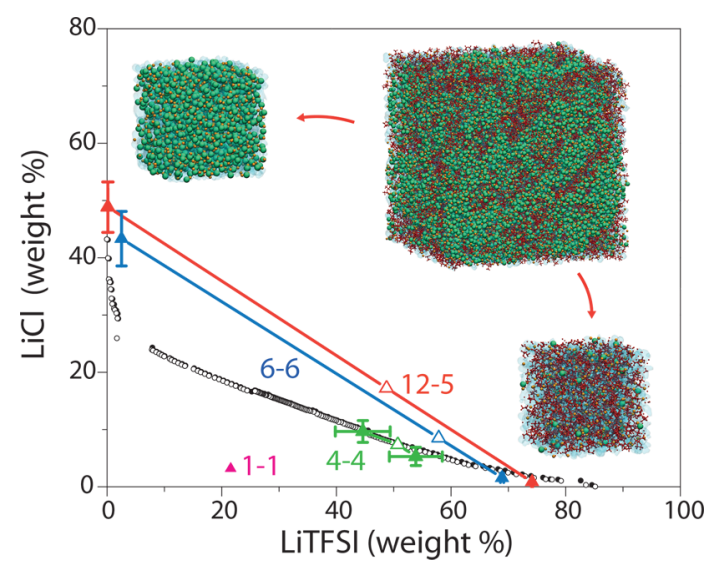

Figure 3. Phase diagram of the ternary $\mathrm{LiCl} / \mathrm{LiTFSI} / \mathrm{H}_{2} \mathrm{O}$ mixture. The phase boundary is located by cloud point experiments; filled black circles correspond to data for a biphasic system, and empty circles correspond to data for a monophasic system. Tie lines are obtained by MD simulations for $12 \mathrm{~m}-5 \mathrm{~m}$ (red), $6 \mathrm{~m}-6 \mathrm{~m}$ (blue), $4 \mathrm{~m}-4 \mathrm{~m}$ (green), and $1 \mathrm{~m}-1 \mathrm{~m}$ (magenta) LiCl-LiTFSI systems and relate the global composition of the system (empty triangles) to that of the resulting phases (filled triangles). MD simulation snapshots illustrate the initial system for the $12 \mathrm{~m} \mathrm{LiCl}-5 \mathrm{~m}$ LiTFSI composition and resulting $\mathrm{LiCl}$ and LiTFSI-rich phases (note that the latter are only close-ups on each phase, because the complete system contains both of them separated by interfaces, as shown in Figure 2B).

respect to the system $(12 \mathrm{~m} \mathrm{LiCl}$ and $5 \mathrm{~m}$ LiTFSI) mentioned above, we also observe a phase separation in the MD simulations for a global composition of $6 \mathrm{~m}$ and $6 \mathrm{~m}$, while for a more dilute system $(1 \mathrm{~m}$ and $1 \mathrm{~m})$, the system remains monophasic (see Figure S2). At a global composition of $4 \mathrm{~m}$ and $4 \mathrm{~m}$, the system is close to the transition between the oneand two-phase behavior (see density profiles for $4 \mathrm{~m}$ and $4 \mathrm{~m}$ and $6 \mathrm{~m}$ and $6 \mathrm{~m}$ in the Supporting Information). The resulting compositions of the coexisting liquids in the phase-separating cases, obtained from the MD density profiles (see the Supporting Information), are very close to the experimental phase boundaries. Such an agreement is remarkable considering the complexity of the system. This further supports the validity of the MD simulations, which in turn complements the experimental phase boundaries with the tie lines (which could in principle be obtained by separating and weighting both phases but would require larger volumes and therefore be much more expensive).

We finally turn to the physical origin of the phase separation. In contrast to previously reported ABSs, in the systems presented here the salts share a common cation. In agreement with previous MD studies of bulk WiSEs, ${ }^{1}$ we find that in our case the relevant cationic species in both phases is the hydrated $\mathrm{Li}^{+}$, despite the observed partial desolvation in this highconcentration regime (see Figure S3). This indicates that the phase separation is essentially driven by the different properties of the anions.
The driving force to form an ABS was previously proposed to result from the difference in interaction with water molecules between two salts, one structuring water (kosmotropic) and the other disordering the hydrogen bond network of water (chaotropic), ${ }^{10}$ a phenomenon classically evidenced by viscosity measurements. ${ }^{13}$ While TFSI $^{-}$was reported to be chaotropic, viscosity measurements carried out in this work (see Figure S4) reveal that $\mathrm{TFSI}^{-}$exhibits a positive JonesDole $B$-coefficient, which is usually observed for kosmotropic salts. This could result from the large size and hydrophobic character of the $\mathrm{TFSI}^{-}$anion. ${ }^{14,15}$ However, the concept of chao- and kosmotropicity, which underlines the effect of individual ions on water, should be taken with some caution at such high concentrations. This is particularly true for WiSEs in which the salt:water ratio is $>1$, because in this regime the effects of ion-water interactions are not additive. ${ }^{16,17}$

From a thermodynamic point of view, a mixture of a solvent with two salts sharing a common ion can be considered as a ternary mixture, due to the constraint of electroneutrality. Using integral equations, Lo Celso et al. analyzed the phase behavior of such mixtures for neutral (solvent) and charged (ions) hard spheres ${ }^{18}$ and found that an asymmetry in the size of the counterions can be sufficient to induce a phase separation, for a range of compositions and temperatures. On the microscopic scale, this means that the liquid structures satisfying both the local electroneutrality and packing constraints with each counterion separately are more stable than for the case in which all ions are mixed. This is not to say that water does not play a role in the mixing free energy, which determines whether the phase separation occurs: It does contribute both energetically (via ion solvation and screening of electrostatic interactions) and entropically (configurational entropy via the composition of each phase). However, this contribution may not be the dominant one. This claim is further supported by the low solubility of alkali halides in conventional ionic liquids. ${ }^{19}$

In this work, we have shown the formation of ABSs formed by mixing salts containing a common cation, namely, LiTFSI and $\mathrm{LiCl}$. With the help of NMR and MD simulations, we demonstrated that for a wide range of compositions, the system segregates into two phases, one being rich in LiTFSI and containing some $\mathrm{LiCl}$ and one containing almost exclusively $\mathrm{LiCl}$ at high concentrations. While the chao- and kosmotropic phenomenological effect of ions was previously suggested, our findings support the idea that the anion size asymmetry is the driving force for the formation of an ABS in this system. While the first ABS-based flow cell battery was proposed recently, ${ }^{20}$ preliminary results indicate the possibility of making use of this $\mathrm{ABS}$ to develop aqueous dual-ion batteries (see Figure S5). Furthermore, controlling this phase separation phenomenon could provide new avenues for recycling LiTFSI from aqueous solutions.

\section{METHODS}

Experimental methods and details of the MD computer simulation are described in the Supporting Information. No unexpected or unusually dangerous safety hazards were encountered. 


\section{ASSOCIATED CONTENT}

\section{S Supporting Information}

The Supporting Information is available free of charge on the ACS Publications website at DOI: 10.1021/acscentsci.8b00955.

Experimental procedures and simulations details, photographs of a drop of LiTFSI in $\mathrm{LiCl}$ aqueous solutions, concentration profiles calculated from MD simulations with different global compositions, solvation of $\mathrm{Li}^{+}$ cations in the $\mathrm{LiCl}$ and LiTFSI-rich phases, viscosity measurements and Jones-Dole coefficients for LiTFSI, $\mathrm{LiCl}$, and $\mathrm{KCl}$ aqueous solutions, and a dual-ion battery using the $\mathrm{ABS}$ as the electrolyte (PDF)

\section{AUTHOR INFORMATION}

\section{Corresponding Authors}

*E-mail: joachim.dzubiella@physik.uni-freiburg.de.

*E-mail: alexis.grimaud@college-de-france.fr.

*E-mail: benjamin.rotenberg@sorbonne-universite.fr.

\section{ORCID}

Nicolas Dubouis: 0000-0001-8698-7202

Chanbum Park: 0000-0002-8613-9278

Matej Kanduč: 0000-0002-5307-7488

Mathieu Salanne: 0000-0002-1753-491X

Joachim Dzubiella: 0000-0001-6751-1487

Alexis Grimaud: 0000-0002-9966-205X

Benjamin Rotenberg: 0000-0001-5198-4650

Notes

The authors declare no competing financial interest.

\section{ACKNOWLEDGMENTS}

B.R. acknowledges financial support from the Alexander von Humboldt foundation via the Bessel research award. N.D. acknowledges Ecole Normale Supérieure for his Ph.D. scholarship. The authors thank the Rechenzentrum of the Albert-Ludwigs-Universität Freiburg and the North-German Supercomputing Alliance (HLRN) for available computer time. A.G. acknowledges financial support from the ANR MIDWAY (Project ANR-17-CE05-0008). This work was also supported by the Ile-de-France Region (DIM RESPORE). M.S. has received funding from the European Research Council (ERC) under the European Union's Horizon 2020 research and innovation program (Grant Agreement 771294). M.D. acknowledges financial support from the French national network "Réseau sur le Stockage Electrochimique de l'Energie" (RS2E) FR CNRS 3459 and from Laboratory of Excellence program STORE-EX (ANR 10-LABX-0076). J.D. and M.K. have received funding from the European Research Council (ERC) under the European Union's Horizon 2020 research and innovation programme (Grant Agreement 646659). M.K. acknowledges financial support from the Slovenian Research Agency (research core funding P1-0055). The authors are grateful to Jean-Pierre Hansen, Olivier Bernard, and Richard Chudoba for useful discussions.

\section{REFERENCES}

(1) Suo, L.; Borodin, O.; Gao, T.; Olguin, M.; Ho, J.; Fan, X.; Luo, C.; Wang, C.; Xu, K. Water-in-Salt" Electrolyte Enables High-Voltage Aqueous Lithium-Ion Chemistries. Science 2015, 350 (6263), 938943.
(2) Yamada, Y.; Usui, K.; Sodeyama, K.; Ko, S.; Tateyama, Y.; Yamada, A. Hydrate-Melt Electrolytes for High-Energy-Density Aqueous Batteries. Nature Energy 2016, 1 (10), 16129.

(3) Suo, L.; Borodin, O.; Sun, W.; Fan, X.; Yang, C.; Wang, F.; Gao, T.; Ma, Z.; Schroeder, M.; von Cresce, A.; et al. Advanced HighVoltage Aqueous Lithium-Ion Battery Enabled by "Water-in-Bisalt" Electrolyte. Angew. Chem., Int. Ed. 2016, 55 (25), 7136-7141.

(4) Dubouis, N.; Lemaire, P.; Mirvaux, B.; Salager, E.; Deschamps, M.; Grimaud, A. The Role of the Hydrogen Evolution Reaction in the Solid-Electrolyte Interphase Formation Mechanism for "Water-inSalt" Electrolytes. Energy Environ. Sci. 2018, 11 (12), 3491-3499.

(5) Visser, A. E.; Swatloski, R. P.; Rogers, R. D. PH-Dependent Partitioning in Room Temperature Ionic Liquids Provides a Link to Traditional Solvent Extraction Behavior. Green Chem. 2000, 2 (1), 14.

(6) Huddleston, J. G.; Willauer, H. D.; Swatloski, R. P.; Visser, A. E.; Rogers, R. D. Room Temperature Ionic Liquids as Novel Media for 'Clean' Liquid-Liquid Extraction. Chem. Commun. 1998, 0 (16), $1765-1766$.

(7) Gras, M.; Papaiconomou, N.; Schaeffer, N.; Chainet, E.; Tedjar, F.; Coutinho, J. A. P.; Billard, I. Ionic-Liquid-Based Acidic Aqueous Biphasic Systems for Simultaneous Leaching and Extraction of Metallic Ions. Angew. Chem., Int. Ed. 2018, 57 (6), 1563-1566.

(8) Beijerinck, M. Über Eine Eigentümlichkeit Der Löslichen Stärke. Zentralbl. Bakteriol., Parasitenkd. Infektionskrankh. 1896, 2, 679-699.

(9) Gutowski, K. E.; Broker, G. A.; Willauer, H. D.; Huddleston, J. G.; Swatloski, R. P.; Holbrey, J. D.; Rogers, R. D. Controlling the Aqueous Miscibility of Ionic Liquids: Aqueous Biphasic Systems of Water-Miscible Ionic Liquids and Water-Structuring Salts for Recycle, Metathesis, and Separations. J. Am. Chem. Soc. 2003, 125 (22), 66326633.

(10) Bridges, N. J.; Gutowski, K. E.; Rogers, R. D. Investigation of Aqueous Biphasic Systems Formed from Solutions of Chaotropic Salts with Kosmotropic Salts (Salt-Salt ABS). Green Chem. 2007, 9 (2), 177-183.

(11) Freire, M. G.; Cláudio, A. F. M.; Araújo, J. M. M.; Coutinho, J. A. P.; Marrucho, I. M.; Lopes, J. N. C.; Rebelo, L. P. N. Aqueous Biphasic Systems: A Boost Brought about by Using Ionic Liquids. Chem. Soc. Rev. 2012, 41 (14), 4966.

(12) Howlett, P. C.; Izgorodina, E. I.; Forsyth, M.; MacFarlane, D. R. Electrochemistry at Negative Potentials in Bis(Trifluoromethanesulfonyl)Amide Ionic Liquids. Z. Phys. Chem. 2006, 220 (10), 1483-1498.

(13) Jenkins, H. D. B.; Marcus, Y. Viscosity B-Coefficients of Ions in Solution. Chem. Rev. 1995, 95 (8), 2695-2724.

(14) Su, H.-L.; Lan, M.-T.; Lin, K.-W.; Hsieh, Y.-Z. Chaotropic Salts: Novel Modifiers for the Capillary Electrophoretic Analysis of Benzodiazepines. Electrophoresis 2008, 29 (16), 3384-3390.

(15) Marcus, Y. Effect of Ions on the Structure of Water: Structure Making and Breaking. Chem. Rev. 2009, 109 (3), 1346-1370.

(16) Borodin, O.; Suo, L.; Gobet, M.; Ren, X.; Wang, F.; Faraone, A.; Peng, J.; Olguin, M.; Schroeder, M.; Ding, M. S.; et al. Liquid Structure with Nano-Heterogeneity Promotes Cationic Transport in Concentrated Electrolytes. ACS Nano 2017, 11 (10), 10462-10471.

(17) Levy, A.; McEldrew, M.; Bazant, M. Z. Spin-Glass Charge Ordering in Ionic Liquids. arXiv 2018, 1808.06156.

(18) Lo Celso, F.; Triolo, A.; Triolo, R. Phase Separation in MultiComponent Mixtures: The Four-Component Case. Phys. A 2002, 304 (1), 299-307.

(19) Kuzmina, O.; Bordes, E.; Schmauck, J.; Hunt, P. A.; Hallett, J. P.; Welton, T. Solubility of Alkali Metal Halides in the Ionic Liquid [C4C1im][OTf]. Phys. Chem. Chem. Phys. 2016, 18 (24), 1616116168.

(20) Navalpotro, P.; Neves, C. M. S. S.; Palma, J.; Freire, M. G.; Coutinho, J. A. P.; Marcilla, R. Pioneering Use of Ionic Liquid-Based Aqueous Biphasic Systems as Membrane-Free Batteries. Advanced Science 2018, 5 (10), 1800576. 